\title{
Complex bend of multilayered concrete rods
}

\author{
Yuri Nemirovskii ${ }^{1, *}$ and Sergey Tikhonov ${ }^{2}$ \\ ${ }^{1}$ Khristianovich Institute of Theoretical and Applied Mechanics Siberian Branch of the Russian \\ Academy of Sciences, Physics of Fast Processes Laboratory, 630090 Institutskaya str., 4/1, Novosi- \\ birsk, Russia \\ ${ }^{2}$ I. Ulianov Chuvash State University, Faculty of Information and Computer Systems, 428015 \\ Moskovskiy pr., 14, Cheboksary, Russia
}

\begin{abstract}
The problem of complex bend of multilayered rods based on concrete is considered. It is assumed that the rod of constant cross-section is of arbitrary shape and different brands of concrete are used in the cross-section of the rod layer by layer. The solution is sought by the small parameter method. The case of a complex bend of the rod pinched at both ends is considered as an example of this solution method. The distribution of bending moments and longitudinal forces in the zero and first approximations is determined.
\end{abstract}

\section{Introduction}

Rectilinear rods of various cross-section are widely used in different branches of mechanical engineering, aircraft and shipbuilding, in civil and industrial construction projects. During operation, they are affected by thermal, chemical and kinematic forces, and due to the production of the same type of serial elements, they are subject to increased requirements for reliable operation in short-term and long-term modes. In modern economic conditions, these requirements cannot be implemented with the use of traditional structural materials and therefore in recent decades, technologies have actively been developed to make heterogeneous and multilayered structures in which materials with different properties can work together to achieve a common goal: long-term and reliable operation at a reasonable cost of its maintenance. The paper deals with structures that belong to the category of layered rods with significantly different properties in the layers, which should be determined by special experiments and taken into account in the developed calculation method. Such method should be sufficiently reliable, relatively simple and flexible to take into account a wide range of possible variations of conditions of fastening, loading and structural forms of sections. The corresponding solutions for structures operating under flat bend conditions are obtained in the works [1], [2], [3], [4]. For complex bend conditions, the corresponding solutions are absent. We will consider concretes as the material of each layer. In calculations, we will take into account that concrete behaves almost as linearly elastic under tension, while under compression the diagrams show significant nonlinearity even at low loading levels.

\footnotetext{
*e-mail: nemirov@itam.nsc.ru
} 


\section{Methods}

As a solution method of the problem, we use the method of a small parameter which showed high efficiency in solving a wide class of problems [5], [6], [7],[8], [9].

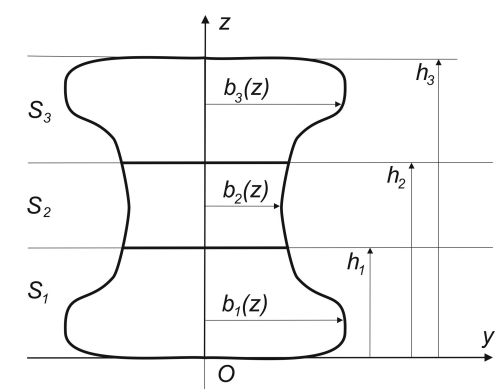

Figure 1. A cross-section of the rod when the number of layers $n=3$

We take the law of deformation for all materials as

$$
\sigma_{i}=A_{i} \varepsilon+B_{i} \varepsilon^{2}
$$

where $A_{i}, B_{i}$ - are coefficients determined from experiments.

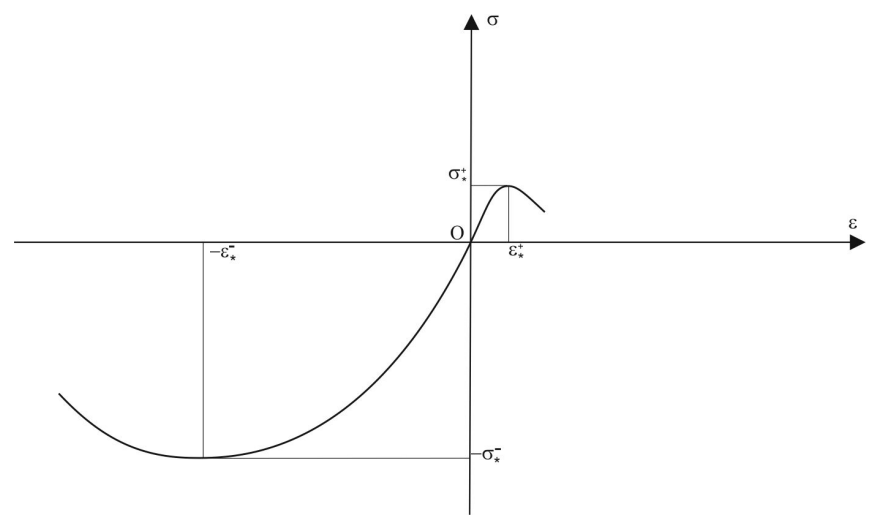

Figure 2. A tension-compression diagram of concrete

The deformation $\varepsilon$ for each layer should not exceed the limit values at tension $\varepsilon_{* i}^{+}$and compression $\varepsilon_{* i}^{-}$(figure 2), i.e. dependencies (1) should be determined on the segment

$$
-\varepsilon_{* i}^{-} \leq \varepsilon \leq \varepsilon_{* i}^{+} .
$$

The ratios [10] obtained from the analysis of the diagram (figure 2) can be used to determine the coefficients $A_{i}, B_{i}$.

We differentiate the ratios (1), then obtain

$$
\frac{d \sigma_{i}}{d \varepsilon}=A_{i}+2 B_{i} \varepsilon
$$

From (2) we obtain passing to the limit 


$$
\left.\frac{d \sigma_{i}}{d \varepsilon}\right|_{\varepsilon \rightarrow 0}=A_{i}
$$

where we get

$$
A_{i}=E_{i}
$$

where $E_{i}$ is an elasticity modulus of concrete of $i$-th layer.

Considering that the point $\left(-\varepsilon_{* i}^{-},-\sigma_{* i}^{-}\right)$in the diagram (figure 2$)$ is the extremum point, we obtain from the ratios (1), (2), (4)

$$
\varepsilon_{* i}^{-}=2 \frac{\sigma_{* i}^{-}}{E}, \quad B_{i}=\frac{1}{4} \frac{E_{i}^{2}}{\sigma_{* i}^{-}} .
$$

Taking into account that the tensile strength $\sigma_{* i}^{+}$is much less than compression strength $\sigma_{* i}^{-}$, we consider the following ratios to be valid

$$
\varepsilon_{* i}^{+}=\frac{\sigma_{* i}^{+}}{E} .
$$

Thus, when using approximations (1) it is enough to have three traditional characteristics $\sigma_{* i}^{+}, \sigma_{* i}^{-}, E$.

If there are diagrams of concrete deformation of each layer of the rod under tension and compression, the coefficients $A_{i}, B_{i}$ in equations (1) can also be obtained by the method of least squares.

Table 2 shows the values of the coefficients of equations (1), obtained by the formulas (4), (5), (6) and the method of least squares for concrete grades B10, B30, B50 on the basis of deformation diagrams obtained in experiments [11].

Table 1. The coefficients of approximating curves $A_{i}, B_{i}$

\begin{tabular}{|c|c|c|c|c|c|c|}
\hline \multirow{2}{*}{ Concrete } & \multicolumn{3}{|c|}{ Method of least squares } & \multicolumn{3}{c|}{ By formulas (4), (5), (6) } \\
\cline { 2 - 7 } & $A_{i}, \mathrm{MPa}$ & $B_{i}, \mathrm{MPa}$ & $R^{2}$ & $A_{i}, \mathrm{MPa}$ & $B_{i}, \mathrm{MPa}$ & $R^{2}$ \\
\hline $\mathrm{B} 10$ & 12488 & 5078524 & 0,96 & 17800 & 10589572 & 0,90 \\
\hline $\mathrm{B} 30$ & 32351 & 11660358 & 0,94 & 32200 & 11890367 & 0,94 \\
\hline B50 & 56490 & 21305730 & 0,92 & 38600 & 10375766 & 0,84 \\
\hline
\end{tabular}

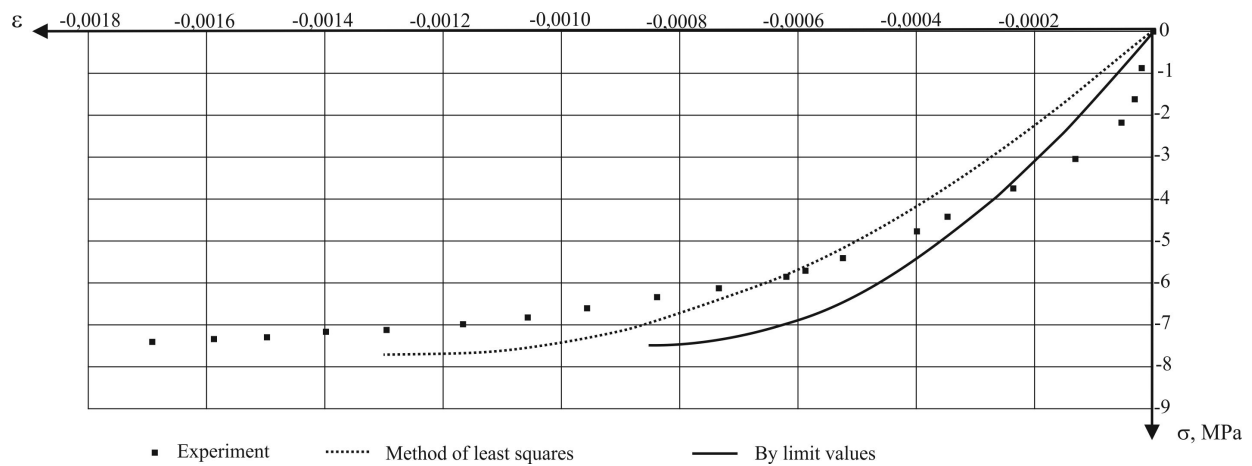

Figure 3. A compression diagram of concrete grade B10 


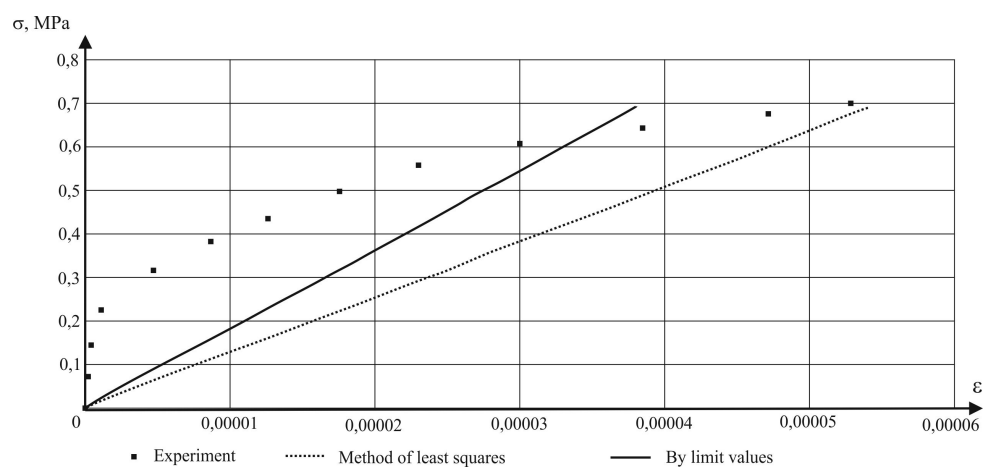

Figure 4. A tension diagram of concrete grade B10

Comparisons of deformation diagrams constructed by approximating formulas (1), the coefficients of which are determined by the method of least squares and by the ratios (4), (5), (6), with experimental diagrams are shown in figures 3, 4. It is visible from the figures that experimental diagrams approach the solution well enough at deformations close to the limit.

We rewrite the deformation condition for $i$-th layer in the form

$$
\sigma_{i}=A_{i}\left(\frac{\varepsilon}{\varepsilon_{* i}}\right)\left(\varepsilon_{* i}+\frac{B_{i}}{A_{i}}\left(\varepsilon_{* i}\right)^{2}\left(\frac{\varepsilon}{\varepsilon_{* i}}\right)\right),
$$

where

$$
\varepsilon_{* i}=\min \left\{\varepsilon_{* i}^{+}, \varepsilon_{* i}^{-}\right\}
$$

To solve this problem, we introduce a small dimensionless parameter $\delta$, which is determined according to the ratio (7) as

$$
\delta=\frac{B_{i}}{A_{i}}\left(\varepsilon_{* i}\right)^{2}
$$

If we determine the parameter $\delta$ at $i=1$ and assume that the first layer of the rod is made of concrete grade $\mathrm{B} 10$, then from 2 we obtain the value of the parameter $\delta \approx 0,0038$.

We rewrite the ratios (7) in the form

$$
\sigma_{i}=a_{i} \varepsilon+c_{i} \delta \varepsilon^{2}
$$

where $a_{i}=A_{i}, c_{i}=\frac{A_{1} B_{i}}{A_{i} B_{1} \varepsilon_{* 1}^{2}}$.

Using the classical kinematic hypotheses of Kirchhoff-Lyav, for deformations we have expressions [12]

$$
\varepsilon(x, y, z)=\varepsilon_{0}(x)-y \kappa_{z}(x)+z \kappa_{y}(x),
$$

where

$$
\varepsilon_{0}(x)=\frac{d u_{0}}{d x}, \quad \kappa_{y}=\frac{d^{2} w_{0}}{d x^{2}}, \quad \kappa_{z}=\frac{d^{2} v_{0}}{d x^{2}}
$$


We consider that the values $\sigma(x, y, z), u_{0}(x), v_{0}(x), w_{0}(x)$ depend on the specified parameter $\delta$. We expand them into series of degrees of the specified parameter

$$
\begin{gathered}
\sigma(x, y, z)=\sum_{k=0}^{\infty} \delta^{k} \sigma^{(k)}(x, y, z), u_{0}(x)=\sum_{k=0}^{\infty} \delta^{k} u_{0}^{(k)}(x), \\
v_{0}(x)=\sum_{k=0}^{\infty} \delta^{k} v_{0}^{(k)}(x), w_{0}(x)=\sum_{k=0}^{\infty} \delta^{k} w_{0}^{(k)}(x),
\end{gathered}
$$

where the components at tension and movements get the index $(n)$, corresponding to the degree of parameter $\delta$.

From ratios (10), (11), (12), we obtain the expression for deformations

$$
\varepsilon(x, y, z)=\sum_{n=0}^{\infty} \delta^{n} \varepsilon^{(n)}(x, y, z), \quad \varepsilon^{(n)}(x, y, z)=\frac{d u_{0}^{(n)}}{d x}+z \frac{d^{2} w_{0}^{(n)}}{d x^{2}}-y \frac{d^{2} v_{0}^{(n)}}{d x^{2}}
$$

Substituting (12), (13) in the expression (9) and equating the ratios at identical degrees of $\delta$, we obtain for the zero and first approximations

$$
\begin{gathered}
\sigma_{(i)}^{(0)}=a_{i} \varepsilon^{(0)}, \\
\sigma_{(i)}^{(1)}=a_{i} \varepsilon^{(1)}+c_{i}\left(\varepsilon^{(0)}\right)^{2} .
\end{gathered}
$$

Longitudinal forces and bending moments can be determined from the ratios

$$
N=\sum_{i=1}^{n} \int_{S_{i}} \sigma_{i} d S, \quad M_{y}=\sum_{i=1}^{n} \int_{S_{i}} \sigma_{i} z d S, \quad M_{z}=-\sum_{i=1}^{n} \int_{S_{i}} \sigma_{i} y d S .
$$

Equilibrium equations have the form [12]

$$
\frac{d^{2} M_{z}}{d x^{2}}=q_{y}-\frac{d m_{z}}{d x}, \quad \frac{d^{2} M_{y}}{d x^{2}}=q_{z}-\frac{d m_{y}}{d x}, \quad \frac{d N}{d x}=-q_{x},
$$

where $N$ is projections of the vector of distributed loading of the rod attached to the axis, $M_{z}, M_{y}$ are projections of the vector of distributed moments on $z$ and $y$ axes.

Substituting the expansion for stresses (16) in (12) we obtain

$$
N=\sum_{n=0}^{\infty} \delta^{n} N^{(n)}, M_{y}=\sum_{n=0}^{\infty} \delta^{n} M_{y}^{(n)}, M_{z}=\sum_{n=0}^{\infty} \delta^{n} M_{z}^{(n)}
$$

where

$$
N^{(i)}=\sum_{j=1}^{n} \int_{S_{j}} \sigma_{j}^{(i)} d S, \quad M_{y}^{(i)}=\sum_{j=1}^{n} \int_{S_{j}} \sigma_{j}^{(i)} z d S, \quad M_{z}^{(i)}=-\sum_{i=1}^{n} \int_{S_{i}} \sigma_{j}^{(i)} y d S .
$$

From equilibrium equations (17) and ratios (18), we obtain for the zero approximation

$$
\frac{d^{2} M_{z}^{(0)}}{d x^{2}}=q_{y}-\frac{d m_{z}}{d x}, \quad \frac{d^{2} M_{y}^{(0)}}{d x^{2}}=q_{z}-\frac{d m_{y}}{d x}, \quad \frac{d N^{(0)}}{d x}=-q_{x} .
$$


From (13), (14), (19) we determine the longitudinal force and bending moments

$$
\begin{array}{r}
N^{(0)}=2 \sum_{i=1}^{n} \iint_{S} \sigma_{i}^{(0)} d S=2 \sum_{i=1}^{n} \int_{h_{i-1}}^{h_{i}} d z \int_{0}^{b_{i}(z)} \sigma_{i}^{(0)} d y=2 \sum_{i=1}^{n} a_{i} \int_{h_{i-1}}^{h_{i}} d z \int_{0}^{b_{i}(z)} \varepsilon^{(0)} d y= \\
=2 \sum_{i=1}^{n} a_{i} \int_{h_{i-1}}^{h_{i}} d z \int_{0}^{b_{i}(z)}\left(\frac{d u_{0}^{(0)}}{d x}-y \frac{d^{2} v_{0}^{(0)}}{d x^{2}}+z \frac{d^{2} w_{0}^{(0)}}{d x^{2}}\right) d y=f_{1} \frac{d u_{0}^{(0)}}{d x}+f_{2} \frac{d^{2} w_{0}^{(0)}}{d x^{2}}+f_{3} \frac{d^{2} w_{0}^{(0)}}{d x^{2}}
\end{array}
$$

where

$$
\begin{gathered}
f_{1}=2 \sum_{i=1}^{n} a_{i} \int_{h_{i-1}}^{h_{i}} b_{i}(z) d z, \quad f_{2}=-\sum_{i=1}^{n} a_{i} \int_{h_{i-1}}^{h_{i}} b_{i}^{2}(z) d z, \quad f_{3}=2 \sum_{i=1}^{n} a_{i} \int_{h_{i-1}}^{h_{i}} z b_{i}(z) d z \\
M_{y}^{(0)}=2 \sum_{i=1}^{n} \iint_{S} z \sigma_{i}^{(0)} d S=2 \sum_{i=1}^{n} a_{i} \int_{h_{i-1}}^{h_{i}} d z \int_{0}^{b_{i}(z)} z \sigma_{i}^{(0)} d y= \\
=2 \sum_{i=1}^{n} a_{i} \int_{h_{i-1}}^{h_{i}} d z \int_{0}^{b_{i}(z)}\left(z \frac{d u_{0}^{(0)}}{d x}-z y \frac{d^{2} v_{0}^{(0)}}{d x^{2}}+z^{2} \frac{d^{2} w_{0}^{(0)}}{d x^{2}}\right) d y=f_{3} \frac{d u_{0}^{(0)}}{d x}+f_{4} \frac{d^{2} v_{0}^{(0)}}{d x^{2}}+f_{5} \frac{d^{2} w_{0}^{(0)}}{d x^{2}}
\end{gathered}
$$

where

$$
\begin{gathered}
f_{4}=-\sum_{i=1}^{n} a_{i} \int_{h_{i-1}}^{h_{i}} z b_{i}^{2}(z) d z, \quad f_{5}=2 \sum_{i=1}^{n} a_{i} \int_{h_{i-1}}^{h_{i}} z^{2} b_{i}(z) d z \\
M_{z}^{(0)}=-2 \sum_{i=1}^{n} \iint_{S} y \sigma_{i}^{(0)} d S=-2 \sum_{i=1}^{n} a_{i} \int_{h_{i-1}}^{h_{i}} d z \int_{0}^{b_{i}(z)} \sigma_{i}^{(0)} y d y= \\
=-2 \sum_{i=1}^{n} a_{i} \int_{h_{i-1}}^{h_{i}} d z \int_{0}^{b_{i}(z)}\left(y \frac{d u_{0}^{(0)}}{d x}+z y \frac{d^{2} v_{0}^{(0)}}{d x^{2}}+y^{2} \frac{d^{2} w_{0}^{(0)}}{d x^{2}}\right) d y=f_{2} \frac{d u_{0}^{(0)}}{d x}+f_{6} \frac{d^{2} v_{0}^{(0)}}{d x^{2}}+f_{4} \frac{d^{2} w_{0}^{(0)}}{d x^{2}}
\end{gathered}
$$

where

$$
f_{6}=\frac{2}{3} \sum_{i=1}^{n} a_{i} \int_{h_{i-1}}^{h_{i}} b_{i}^{3}(z) d z .
$$

From (17), (21) we obtain a system of linear differential equations to determine the movements in the zero approximation

$$
\begin{gathered}
f_{4} \frac{d^{4} w_{0}^{(0)}}{d x^{4}}+f_{6} \frac{d^{4} v_{0}^{(0)}}{d x^{4}}+f_{2} \frac{d^{3} u_{0}^{(0)}}{d x^{3}}=q_{y}-\frac{d m_{z}}{d x}, \\
f_{5} \frac{d^{4} w_{0}^{(0)}}{d x^{4}}+f_{4} \frac{d^{4} v_{0}^{(0)}}{d x^{4}}+f_{3} \frac{d^{3} u_{0}^{(0)}}{d x^{3}}=q_{z}-\frac{d m_{y}}{d x}, \\
f_{3} \frac{d^{3} w_{0}^{(0)}}{d x^{3}}+f_{2} \frac{d^{3} v_{0}^{(0)}}{d x^{3}}+f_{1} \frac{d^{2} u_{0}^{(0)}}{d x^{2}}=-q_{x} .
\end{gathered}
$$


From the last equation of the system (22), we express $\frac{d^{2} u_{0}^{(0)}}{d x^{2}}$ and substitute in the first two. We obtain a system of two equations and two unknown functions

$$
\begin{gathered}
g_{1} \frac{d^{4} w_{0}^{(0)}}{d x^{4}}+g_{2} \frac{d^{4} v_{0}^{(0)}}{d x^{4}}=f_{2} \frac{d q_{x}}{d x}-f_{1} \frac{d m_{z}}{d x}+f_{1} q_{y}, \\
g_{3} \frac{d^{4} w_{0}^{(0)}}{d x^{4}}+g_{1} \frac{d^{4} v_{0}^{(0)}}{d x^{4}}=f_{3} \frac{d q_{x}}{d x}-f_{1} \frac{d m_{y}}{d x}+f_{1} q_{z},
\end{gathered}
$$

where

$$
g_{1}=f_{4} f_{1}-f_{3} f_{2}, \quad g_{2}=f_{6} f_{1}+f_{2}^{2}, \quad g_{3}=f_{5} f_{1}-f_{3}^{2} .
$$

From (23) we obtain

$$
\begin{aligned}
& \frac{d^{4} w_{0}^{(0)}}{d x^{4}}=g_{4} \frac{d q_{x}}{d x}+g_{5} \frac{d m_{z}}{d x}+g_{6} \frac{d m_{y}}{d x}-g_{5} q_{y}-g_{6} q_{z}, \\
& \frac{d^{4} v_{0}^{(0)}}{d x^{4}}=g_{7} \frac{d q_{x}}{d x}+g_{8} \frac{d m_{z}}{d x}+g_{9} \frac{d m_{y}}{d x}-g_{8} q_{y}-g_{9} q_{z},
\end{aligned}
$$

where

$$
\begin{gathered}
g_{4}=\frac{f_{2} g_{1}-f_{3} g_{2}}{g_{1}^{2}-g_{2} g_{3}}, g_{5}=-\frac{f_{1} g_{1}}{g_{1}^{2}-g_{2} g_{3}}, \quad g_{6}=\frac{f_{1} g_{2}}{g_{1}^{2}-g_{2} g_{3}}, \\
g_{7}=\frac{f_{2} g_{3}-f_{3} g_{1}}{g_{2} g_{3}-g_{1}^{2}}, \quad g_{8}=-\frac{f_{1} g_{3}}{g_{2} g_{3}-g_{1}^{2}}, \quad g_{9}=\frac{f_{1} g_{1}}{g_{2} g_{3}-g_{1}^{2}} .
\end{gathered}
$$

Where we get from (24), (25)

$$
\begin{aligned}
& w_{0}^{(0)}=\int_{0}^{x} d x \int_{0}^{x} d x \int_{0}^{x} d x \int_{0}^{x}\left(g_{4} \frac{d q_{x}}{d x}+g_{5} \frac{d m_{z}}{d x}+g_{6} \frac{d m_{y}}{d x}-g_{5} q_{y}-g_{6} q_{z}\right) d x+ \\
& +C_{1} x^{3}+C_{2} x^{2}+C_{3} x+C_{4}, \\
& v_{0}^{(0)}=\int_{0}^{x} d x \int_{0}^{x} d x \int_{0}^{x} d x \int_{0}^{x}\left(g_{7} \frac{d q_{x}}{d x}+g_{8} \frac{d m_{z}}{d x}+g_{9} \frac{d m_{y}}{d x}-g_{8} q_{y}-g_{9} q_{z}\right) d x+ \\
& +C_{5} x^{3}+C_{6} x^{2}+C_{7} x+C_{8},
\end{aligned}
$$

where the values of constants $C_{1}, C_{2}, C_{3}, C_{4}, C_{5}, C_{6}, C_{7}, C_{8}$ can be determined from fastening conditions of the rod.

From the last equation of the system (22), we obtain

$$
\frac{d^{2} u_{0}^{(0)}}{d x^{2}}=-\frac{q_{x}}{f_{1}}-\frac{f_{3}}{f_{1}} \frac{d^{3} w_{0}^{(0)}}{d x^{3}}-\frac{f_{2}}{f_{1}} \frac{d^{3} v_{0}^{(0)}}{d x^{3}} .
$$

We substitute the found values $v_{0}^{(0)}, w_{0}^{(0)}$ into expression (26), and from the fastening conditions we get the expression for $u_{0}^{(0)}$. Then we can obtain an expression for deformations in the zero approximation

$$
\varepsilon^{(0)}(x, y, z)=\frac{d u_{0}^{(0)}}{d x}+z \frac{d^{2} w_{0}^{(0)}}{d x^{2}}-y \frac{d^{2} v_{0}^{(0)}}{d x^{2}} .
$$


We substitute the obtained value (27) in (15) and have

$$
\begin{aligned}
& \sigma_{(i)}^{(1)}=a_{i}\left(\frac{d u_{0}^{(1)}}{d x}-y \frac{d^{2} v_{0}^{(1)}}{d x^{2}}+z \frac{d^{2} w_{0}^{(1)}}{d x^{2}}\right)+c_{i}\left(\left(\frac{d u_{0}^{(0)}}{d x}\right)^{2}+y^{2}\left(\frac{d v_{0}^{(0)}}{d x}\right)^{2}+\right. \\
& \left.+z^{2}\left(\frac{d w_{0}^{(0)}}{d x}\right)^{2}-2 y \frac{d u_{0}^{(0)}}{d x} \frac{d^{2} v_{0}^{(0)}}{d x^{2}}+2 z \frac{d u_{0}^{(0)}}{d x} \frac{d^{2} w_{0}^{(0)}}{d x^{2}}-2 z y \frac{d^{2} v_{0}^{(0)}}{d x^{2}} \frac{d^{2} w_{0}^{(0)}}{d x^{2}}\right) .
\end{aligned}
$$

We define the longitudinal force and bending moments in the first approximation

$$
\begin{gathered}
N^{(1)}=2 \sum_{i=1}^{n} \int_{h_{i-1}}^{h_{i}} d z \int_{0}^{b_{i}(z)} \sigma_{i}^{(1)} d y=f_{1} \frac{d u_{0}^{(1)}}{d x}+f_{2} \frac{d^{2} v_{0}^{(1)}}{d x^{2}}+f_{3} \frac{d^{2} w_{0}^{(1)}}{d x^{2}}+j_{1}(x) \\
j_{1}(x)=r_{1}\left(\frac{d u_{0}^{(0)}}{d x}\right)^{2}+r_{4}\left(\frac{d^{2} v_{0}^{(0)}}{d x^{2}}\right)^{2}+r_{6}\left(\frac{d^{2} w_{0}^{(0)}}{d x^{2}}\right)^{2}+2 r_{2} \frac{d u_{0}^{(0)}}{d x} \frac{d^{2} v_{0}^{(0)}}{d x^{2}}+ \\
+2 r_{5} \frac{d^{2} v_{0}^{(0)}}{d x^{2}} \frac{d^{2} w_{0}^{(0)}}{d x^{2}}+2 r_{3} \frac{d u_{0}^{(0)}}{d x} \frac{d^{2} w_{0}^{(0)}}{d x^{2}}
\end{gathered}
$$

where

$$
\begin{gathered}
r_{1}=2 \sum_{i=1}^{n} c_{i} \int_{h_{i-1}}^{h_{i}} b_{i}(z) d z, \quad r_{2}=-\sum_{i=1}^{n} c_{i} \int_{h_{i-1}}^{h_{i}} b_{i}^{2}(z) d z, \quad r_{3}=2 \sum_{i=1}^{n} c_{i} \int_{h_{i-1}}^{h_{i}} z b_{i}(z) d z \\
r_{4}=\frac{2}{3} \sum_{i=1}^{n} c_{i} \int_{h_{i-1}}^{h_{i}} b_{i}^{3}(z) d z, \quad r_{5}=-\sum_{i=1}^{n} c_{i} \int_{h_{i-1}}^{h_{i}} z b_{i}^{2}(z) d z, \quad r_{6}=2 \sum_{i=1}^{n} c_{i} \int_{h_{i-1}}^{h_{i}} z^{2} b_{i}(z) d z . \\
M_{y}^{(1)}=2 \sum_{i=1}^{n} \int_{h_{i-1}}^{h_{i}} d z \int_{0}^{b_{i}(z)} z \sigma_{i}^{(1)} d y=f_{3} \frac{d u_{0}^{(1)}}{d x}+f_{4} \frac{d^{2} v_{0}^{(1)}}{d x^{2}}+f_{5} \frac{d^{2} w_{0}^{(1)}}{d x^{2}}+j_{2}(x), \\
j_{2}(x)=r_{3}\left(\frac{d u_{0}^{(0)}}{d x}\right)^{2}+r_{7}\left(\frac{d^{2} v_{0}^{(0)}}{d x^{2}}\right)^{2}+r_{8}\left(\frac{d^{2} w_{0}^{(0)}}{d x^{2}}\right)^{2}+2 r_{5} \frac{d u_{0}^{(0)}}{d x} \frac{d^{2} v_{0}^{(0)}}{d x^{2}}+ \\
+r_{9} \frac{d^{2} v_{0}^{(0)}}{d x^{2}} \frac{d^{2} w_{0}^{(0)}}{d x^{2}}+2 r_{6} \frac{d u_{0}^{(0)}}{d x} \frac{d^{2} w_{0}^{(0)}}{d x^{2}},
\end{gathered}
$$

where

$$
\begin{gathered}
r_{7}=\frac{2}{3} \sum_{i=1}^{n} c_{i} \int_{h_{i-1}}^{h_{i}} z b_{i}^{3}(z) d z, \quad r_{8}=2 \sum_{i=1}^{n} c_{i} \int_{h_{i-1}}^{h_{i}} z^{3} b_{i}(z) d z, \quad r_{9}=-2 \sum_{i=1}^{n} c_{i} \int_{h_{i-1}}^{h_{i}} z^{2} b_{i}^{2}(z) d z . \\
M_{z}^{(1)}=-2 \sum_{i=1}^{n} \int_{h_{i-1}}^{h_{i}} d z \int_{0}^{b_{i}(z)} \sigma_{i}^{(1)} y d y=f_{2} \frac{d u_{0}^{(1)}}{d x}+f_{5} \frac{d^{2} v_{0}^{(1)}}{d x^{2}}+f_{6} \frac{d^{2} w_{0}^{(1)}}{d x^{2}}+j_{3}(x)
\end{gathered}
$$

where

$$
j_{3}(x)=r_{2}\left(\frac{d u_{0}^{(0)}}{d x}\right)^{2}+r_{10}\left(\frac{d^{2} v_{0}^{(0)}}{d x^{2}}\right)^{2}+\frac{1}{2} r_{9}\left(\frac{d^{2} w_{0}^{(0)}}{d x^{2}}\right)^{2}+2 r_{4} \frac{d u_{0}^{(0)}}{d x} \frac{d^{2} v_{0}^{(0)}}{d x^{2}}+
$$




$$
+2 r_{7} \frac{d^{2} v_{0}^{(0)}}{d x^{2}} \frac{d^{2} w_{0}^{(0)}}{d x^{2}}+2 r_{5} \frac{d u_{0}^{(0)}}{d x} \frac{d^{2} w_{0}^{(0)}}{d x^{2}}
$$

where

$$
r_{10}=-\frac{1}{2} \sum_{i=1}^{n} c_{i} \int_{h_{i-1}}^{h_{i}} b_{i}^{4}(z) d z .
$$

We substitute the obtained values (29), (30), (31) in equilibrium equations

$$
\frac{d^{2} M_{z}^{(1)}}{d x^{2}}=0, \quad \frac{d^{2} M_{y}^{(1)}}{d x^{2}}=0, \quad \frac{d N^{(1)}}{d x}=0,
$$

and receive a system of three differential equations to determine the movements

$$
\begin{aligned}
& f_{1} \frac{d^{2} u_{0}^{(1)}}{d x^{2}}+f_{2} \frac{d^{3} v_{0}^{(1)}}{d x^{3}}+f_{3} \frac{d^{3} w_{0}^{(1)}}{d x^{3}}+\frac{d j_{1}(x)}{d x}=0, \\
& f_{2} \frac{d^{3} u_{0}^{(1)}}{d x^{3}}+f_{4} \frac{d^{4} v_{0}^{(1)}}{d x^{4}}+f_{5} \frac{d^{4} w_{0}^{(1)}}{d x^{4}}+\frac{d^{2} j_{2}(x)}{d x^{2}}=0, \\
& f_{3} \frac{d^{3} u_{0}^{(1)}}{d x^{3}}+f_{5} \frac{d^{4} v_{0}^{(1)}}{d x^{4}}+f_{6} \frac{d^{4} w_{0}^{(1)}}{d x^{4}}+\frac{d^{2} j_{3}(x)}{d x^{2}}=0 .
\end{aligned}
$$

We rewrite the system (32) as

$$
\begin{gathered}
\frac{d^{2} u_{0}^{(1)}}{d x^{2}}=-\frac{f_{2}}{f_{1}} \frac{d^{3} v_{0}^{(1)}}{d x^{3}}-\frac{f_{3}}{f_{1}} \frac{d^{3} w_{0}^{(1)}}{d x^{3}}-\frac{1}{f_{1}} \frac{d j_{1}(x)}{d x}, \\
\left(f_{4}-\frac{f_{2} f_{3}}{f_{1}}\right) \frac{d^{4} v_{0}^{(1)}}{d x^{4}}+\left(f_{5}-\frac{f_{3}^{2}}{f_{1}}\right) \frac{d^{4} w_{0}^{(1)}}{d x^{4}}+\frac{d^{2} j_{2}(x)}{d x^{2}}-\frac{f_{3}}{f_{1}} \frac{d^{2} j_{1}(x)}{d x^{2}}=0, \\
\left(f_{5}-\frac{f_{2}^{2}}{f_{1}}\right) \frac{d^{4} v_{0}^{(1)}}{d x^{4}}+\left(f_{6}-\frac{f_{3} f_{2}}{f_{1}}\right) \frac{d^{4} w_{0}^{(1)}}{d x^{4}}+\frac{d^{2} j_{3}(x)}{d x^{2}}-\frac{f_{2}}{f_{1}} \frac{d^{2} j_{1}(x)}{d x^{2}}=0,
\end{gathered}
$$

where we get the expressions to determine the movements in the first approximation

$$
\begin{aligned}
\frac{d^{4} v_{0}^{(1)}}{d x^{4}} & =r_{12} \frac{d^{2} j_{1}(x)}{d x^{2}}+r_{13} \frac{d^{2} j_{2}(x)}{d x^{2}}+r_{14} \frac{d^{2} j_{3}(x)}{d x^{2}}=0, \\
\frac{d^{4} w_{0}^{(1)}}{d x^{4}} & =r_{15} \frac{d^{2} j_{1}(x)}{d x^{2}}+r_{16} \frac{d^{2} j_{2}(x)}{d x^{2}}+r_{17} \frac{d^{2} j_{3}(x)}{d x^{2}}=0, \\
\frac{d^{2} u_{0}^{(1)}}{d x^{2}}=- & -\left(\frac{f_{2} r_{12}+f_{3} r_{15}}{f_{1}}\right) \frac{d^{5} j_{1}(x)}{d x^{5}}-\left(\frac{f_{2} r_{13}+f_{3} r_{16}}{f_{1}}\right) \frac{d^{5} j_{2}(x)}{d x^{5}}- \\
& -\left(\frac{f_{2} r_{14}+f_{3} r_{17}}{f_{1}}\right) \frac{d^{5} j_{3}(x)}{d x^{5}}-\frac{1}{f_{1}} \frac{d j_{1}(x)}{d x},
\end{aligned}
$$

where

$$
\begin{gathered}
r_{11}=\left(f_{1} f_{5}-f_{3}^{2}\right)\left(f_{1} f_{5}-f_{2}^{2}\right)-\left(f_{4} f_{1}-f_{2} f_{3}\right)\left(f_{1} f_{6}-f_{2} f_{3}\right), \\
r_{12}=\frac{f_{1} f_{3} f_{5}-f_{1} f_{2} f_{6}}{r_{11}}, \quad r_{13}=\frac{\left(f_{1} f_{6}-f_{2} f_{3}\right) f_{1}}{r_{11}}, \quad r_{14}=\frac{\left(f_{3}^{2}-f_{1} f_{5}\right) f_{1}}{r_{11}}, \\
r_{15}=\frac{f_{5} f_{1} f_{2}-f_{1} f_{3} f_{4}}{r_{11}}, \quad r_{16}=\frac{f_{1} f_{3}^{2}-f_{5} f_{1}^{2}}{r_{11}}, \quad r_{17}=\frac{f_{4} f_{1}^{2}-f_{1} f_{2} f_{3}}{r_{11}} .
\end{gathered}
$$


Having integrated the equations (34), (35), (36) and defined the integration constants from fastening conditions of the rod, we can determine the movements in the first approximation.

From equations (29), (30), (31), we can find the expressions for longitudinal force and bending moments in the first approximation.

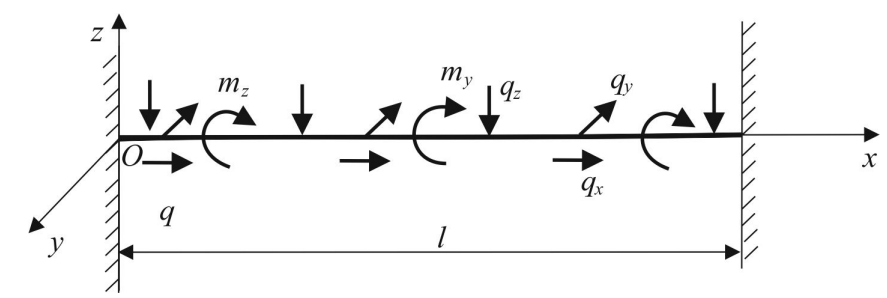

Figure 5. A rod pinched at both ends

\section{Results}

As an example, we consider the problem of complex bend of a rod pinched at both ends (figure 5) by distributed forces and moments

$$
\begin{aligned}
q_{x}(x)=q_{0 x} \frac{x}{l}, & q_{y}(x)=q_{0 y}\left(\alpha_{1}+\alpha_{2} x\right), q_{z}(x)=q_{0 z}\left(\beta_{1}+\beta_{2} x\right), \\
m_{y}(x) & =m_{0 y} \frac{x}{l}, m_{z}(x)=m_{0 z} \frac{x}{l}
\end{aligned}
$$

where $q_{0 x}, q_{0 z}, m_{0}, \alpha_{1}, \alpha_{2}$ are constants.

We assume that the cross-section of the rod has the form figure 6 .

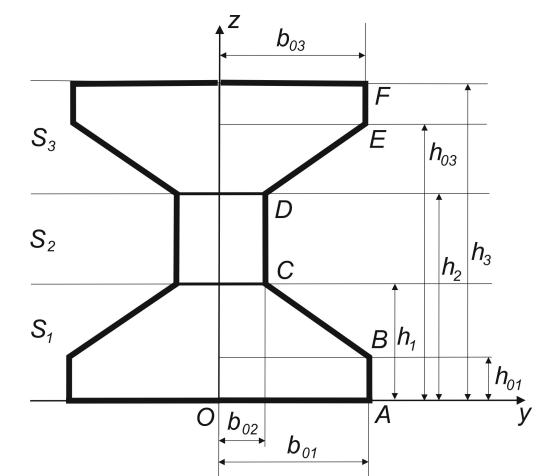

Figure 6. A cross-section of the rod

Coordinates of the points, according to figure 4 have the form $A\left(b_{01}, 0\right), B\left(b_{01}, h_{01}\right)$, $C\left(b_{02}, h_{1}\right), D\left(b_{02}, h_{2}\right), E\left(b_{03}, h_{03}\right), F\left(b_{03}, h_{3}\right)$.

Then the equations of the direct line $B C$ have the form

$$
y=\frac{b_{02}-b_{01}}{h_{1}-h_{01}} z+\frac{b_{01} h_{1}-b_{02} h_{01}}{h_{1}-h_{01}}
$$

for the direct line $D E$

$$
y=\frac{b_{03}-b_{02}}{h_{03}-h_{2}} z+\frac{b_{02} h_{03}-b_{03} h_{2}}{h_{03}-h_{2}} .
$$


Then we get

$$
\begin{aligned}
& f_{1}=\left(\left(h_{1}-h_{01}\right) b_{02}+\left(h_{01}+h_{1}\right) b_{01}\right) a_{1}+\left(-2 h_{1}+2 h_{2}\right) b_{02} a_{2}+ \\
& +\left(\left(h_{03}-h_{2}\right) b_{02}-b_{03}\left(h_{03}+h_{2}-2 h_{3}\right)\right) a_{3} \text {, } \\
& f_{2}=\left(-\frac{1}{3}\left(-h_{01}+h 1\right) b_{02}^{2}+\frac{1}{3} b_{01}\left(h_{01}-h_{1}\right) b_{02}-\frac{2}{3} b_{01}^{2}\left(h_{01}+\frac{1}{2} h_{1}\right)\right) a_{1}+ \\
& +\left(h_{1}-h_{2}\right) b_{02}^{2} a_{2}-\frac{1}{3}\left(\left(h_{03}-h_{2}\right) b_{02}^{2}+\frac{1}{3} b_{03}\left(h_{03}-h_{2}\right) b_{02}+\right. \\
& \left.+\frac{2}{3}\left(h_{03}+\frac{1}{2} h_{2}-\frac{3}{2} h_{3}\right) b_{03}^{2}\right) a_{3}, \\
& f_{3}=\left(-\frac{1}{3}\left(h_{01}+2 h_{1}\right)\left(h_{01}-h_{1}\right) b_{02}+\frac{1}{3} b_{01}\left(h_{01}^{2}+h_{01} h_{1}+h_{1}^{2}\right)\right) a_{1}+ \\
& +\frac{1}{3}\left(-3 h_{1}^{2}+3 h_{2}^{2}\right) b_{02} a_{2}+\frac{1}{3}\left(\left(h_{03}+2 h_{2}\right)\left(h_{03}-h_{2}\right) b_{02}-\right. \\
& \left.-b_{03}\left(h_{03}^{2}+h_{03} h_{2}+h_{2}^{2}-3 h_{3}^{2}\right)\right) a_{3}, \\
& f_{4}=\left(\frac{1}{12} b_{02}^{2}\left(h_{01}+3 h_{1}\right)\right)\left(h_{01}-h_{1}\right)-\frac{1}{6} b_{02} b_{01}\left(-h_{01}^{2}+h_{1}^{2}\right)- \\
& \left.\left.-\frac{1}{4} b_{01}^{2}\left(h_{01}^{2}+\frac{2}{3} h_{01} h_{1}+\frac{1}{3} h_{1}^{2}\right)\right) a_{1}+\frac{1}{2}\left(h_{1}^{2}-h_{2}^{2}\right)\right) b_{02}^{2} a_{2}-\left(\frac{1}{12}\left(h_{03}+3 h_{2}\right)\left(h_{03}-h_{2}\right) b_{02}^{2}+\right. \\
& \left.\frac{1}{12}\left(2 h_{03}^{2}-2 h_{2}^{2}\right) b_{03} b_{02}-\frac{1}{4} b_{03}^{2}\left(h_{03}^{2}+\frac{2}{3} h_{03} h_{2}+\frac{1}{3} h_{2}^{2}-2 h_{3}^{2}\right)\right) a_{3}, \\
& f_{5}=\frac{1}{6}\left(b_{02}\left(-h_{01}^{3}-h_{1} h_{01}^{2}-h_{01} h_{1}^{2}+3 h_{1}^{3}\right)+b_{01}\left(h_{01}+h_{1}\right)\left(h_{01}^{2}+h_{1}^{2}\right)\right) a_{1}+ \\
& +\frac{2}{3} b_{02}\left(-h_{1}^{3}+h_{2}^{3}\right) a_{2}+\frac{1}{6}\left(\left(h_{03}-h_{2}\right)\left(h_{03}^{2}+2 h_{03} h_{2}+3 h_{2}^{2}\right) b_{02}-\right. \\
& \left.-\left(h_{03}^{3}+h_{03}^{2} h_{2}+h_{03} h_{2}^{2}+h_{2}^{3}-4 h_{3}^{3}\right) b_{03}\right) a_{3} \text {, } \\
& f_{6}=\left(\frac{1}{6}\left(h_{1}-h_{01}\right) b_{02}^{3}-\frac{1}{6} b_{01}\left(h_{01}-h_{1}\right) b_{02}^{2}-\frac{1}{6} b_{01}^{2}\left(h_{01}-h_{1}\right) b_{02}+\frac{1}{2}\left(h_{01}+\frac{1}{3} h_{1}\right) b_{01}^{3}\right) a_{1}+ \\
& +\frac{2}{3}\left(h_{2}-h_{1}\right) b_{02}^{3} a_{2}+\left(\frac{1}{6}\left(h_{03}-h_{2}\right) b_{02}^{3}+\frac{1}{6} b_{03}\left(h_{03}-h_{2}\right) b_{02}^{2}+\right. \\
& \left.+\frac{1}{6} b_{03}^{2}\left(h_{03}-h_{2}\right) b_{02}-\frac{1}{6}\left(3 h_{03}+h_{2}-4 h_{3}\right) b_{03}^{3}\right) a_{3}, \\
& r_{1}=\left(\left(h_{1}-h_{01}\right) b_{02}+\left(h_{01}+h_{1}\right) b_{01}\right) c_{1}+\left(-2 h_{1}+2 h_{2}\right) b_{02} c_{2}+ \\
& +\left(\left(h_{03}-h_{2}\right) b_{02}-b_{03}\left(h_{03}+h_{2}-2 h_{3}\right)\right) c_{3} \text {, } \\
& r_{2}=\left(\frac{1}{3}\left(-h_{01}+h 1\right) b_{02}^{2}-\frac{1}{3} b_{01}\left(h_{01}-h_{1}\right) b_{02}+\frac{2}{3} b_{01}^{2}\left(h_{01}+\frac{1}{2} h_{1}\right)\right) c_{1}+ \\
& +\left(-h_{1}+h_{2}\right) b_{02}^{2} c_{2}+\frac{1}{3}\left(\left(h_{03}-h_{2}\right) b_{02}^{2}-\frac{1}{3} b_{03}\left(h_{03}-h_{2}\right) b_{02}-\right. \\
& \left.-\frac{2}{3}\left(h_{03}+\frac{1}{2} h_{2}-\frac{3}{2} h_{3}\right) b_{03}^{2}\right) c_{3} \\
& r_{3}=\left(\frac{1}{3}\left(h_{01}+2 h_{1}\right)\left(h_{01}-h_{1}\right) b_{02}-\frac{1}{3} b_{01}\left(h_{01}^{2}+h_{01} h_{1}+h_{1}^{2}\right)\right) c_{1}-
\end{aligned}
$$




$$
\begin{aligned}
& -\frac{1}{3}\left(-3 h_{1}^{2}+3 h_{2}^{2}\right) b_{02} c_{2}-\frac{1}{3}\left(\left(h_{03}+2 h_{2}\right)\left(h_{03}-h_{2}\right) b_{02}+\right. \\
& \left.+b_{03}\left(h_{03}^{2}+h_{03} h_{2}+h_{2}^{2}-3 h_{3}^{2}\right)\right) c_{3}, \\
& r_{4}=\left(\frac{1}{6}\left(h_{1}-h_{01}\right) b_{02}^{3}-\frac{1}{6} b_{01}\left(h_{01}-h_{1}\right) b_{02}^{2}-\frac{1}{6} b_{01}^{2}\left(h_{01}-h_{1}\right) b_{02}+\right. \\
& \left.+\frac{1}{2}\left(h_{01}+\frac{1}{3} h_{1}\right) b_{01}^{3}\right) c_{1}+\frac{2}{3}\left(h_{2}-h_{1}\right) b_{02}^{3} c_{2}+\left(\frac{1}{6}\left(h_{03}-h_{2}\right) b_{02}^{3}+\frac{1}{6} b_{03}\left(h_{03}-h_{2}\right) b_{02}^{2}+\right. \\
& \left.+\frac{1}{6} b_{03}^{2}\left(h_{03}-h_{2}\right) b_{02}-\frac{1}{6}\left(3 h_{03}+h_{2}-4 h_{3}\right) b_{03}^{3}\right) c_{3} \text {, } \\
& r_{5}=\left(\frac{1}{12} b_{02}^{2}\left(h_{01}+3 h_{1}\right)\right)\left(h_{01}-h_{1}\right)-\frac{1}{6} b_{02} b_{01}\left(-h_{01}^{2}+h_{1}^{2}\right)- \\
& \left.\left.-\frac{1}{4} b_{01}^{2}\left(h_{01}^{2}+\frac{2}{3} h_{01} h_{1}+\frac{1}{3} h_{1}^{2}\right)\right) c_{1}-\frac{1}{2}\left(-h_{1}^{2}+h_{2}^{2}\right)\right) b_{02}^{2} c_{2}-\left(\frac{1}{12}\left(h_{03}+3 h_{2}\right)\left(h_{03}-h_{2}\right) b_{02}^{2}+\right. \\
& \left.+\frac{1}{12}\left(2 h_{03}^{2}-2 h_{2}^{2}\right) b_{03} b_{02}-\frac{1}{4} b_{03}^{2}\left(h_{03}^{2}+\frac{2}{3} h_{03} h_{2}+\frac{1}{3} h_{2}^{2}-2 h_{3}^{2}\right)\right) c_{3}, \\
& r_{6}=\frac{1}{6}\left(b_{02}\left(-h_{01}^{3}-h_{1} h_{01}^{2}-h_{01} h_{1}^{2}+3 h_{1}^{3}\right)+b_{01}\left(h_{01}+h_{1}\right)\left(h_{01}^{2}+h_{1}^{2}\right)\right) c_{1}+ \\
& +\frac{2}{3} b_{02}\left(-h_{1}^{3}+h_{2}^{3}\right) c_{2}+\frac{1}{6}\left(\left(h_{03}-h_{2}\right)\left(h_{03}^{2}+2 h_{03} h_{2}+3 h_{2}^{2}\right) b_{02}-\right. \\
& \left.-\left(h_{03}^{3}+h_{03}^{2} h_{2}+h_{03} h_{2}^{2}+h_{2}^{3}-4 h_{3}^{3}\right) b_{03}\right) c_{3} \text {, } \\
& r_{7}=\left(-\frac{1}{30}\left(h_{01}+4 h_{1}\right)\left(h_{01}-h_{1}\right) b_{02}^{3}-\frac{1}{15}\left(h_{01}+\frac{3}{2} h_{1}\right)\left(h_{01}-h_{1}\right) b_{01} b_{02}^{2}-\right. \\
& \left.-\frac{1}{10}\left(h_{01}-h_{1}\right) b_{01}^{2}\left(h_{01}+\frac{2}{3} h_{1}\right) b_{02}+\frac{1}{5} b_{01}^{3}\left(h_{01}^{2}+\frac{1}{2} h_{01} h_{1}+\frac{1}{6} h_{1}^{2}\right)\right) c_{1}+ \\
& +\frac{1}{3}\left(h_{2}^{2}-h_{1}^{2}\right) b_{02}^{3} c_{2}+\left(\frac{1}{30}\left(h_{03}+4 h_{2}\right)\left(h_{03}-h_{2}\right) b_{02}^{3}+\frac{1}{15}\left(h_{03}+\frac{3}{2} h_{2}\right)\left(h_{03}-h_{2}\right) b_{02}^{2} b_{03}+\right. \\
& \left.+\frac{1}{10} b_{03}^{2} b_{02}\left(h_{03}-h_{2}\right)\left(h_{03}+\frac{2}{3} h_{2}\right)-\frac{1}{5}\left(h_{03}^{2}+\frac{1}{2} h_{03} h_{2}+\frac{1}{6} h_{2}^{2}-\frac{5}{3} h_{3}^{2}\right) b_{03}^{3}\right) c_{3}, \\
& r_{8}=\frac{1}{10}\left(\left(-h_{01}^{4}-h_{01}^{3} h_{1}-h_{01}^{2} h_{1}^{2}-h_{01} h_{1}^{3}+4 h_{1}^{4}\right) b_{02}+\right. \\
& \left.+\left(h_{01}^{4}+h_{01}^{3} h_{1}+h_{01}^{2} h_{1}^{2}+h_{01} h_{1}^{3}+h_{1}^{4}\right) b_{01}\right) c_{1}+ \\
& +\frac{1}{2}\left(h_{2}^{4}-h_{1}^{4}\right) b_{02} c_{2}+\left(\frac{1}{10}\left(h_{03}-h_{2}\right)\left(h_{03}^{3}+2 h_{03}^{2} h_{2}+3 h_{03} h_{2}^{2}+4 h_{2}^{3}\right) b_{02}-\right. \\
& \left.-\frac{1}{10} b_{03}\left(h_{03}^{4}+h_{03}^{3} h_{2}+h_{03}^{2} h_{2}^{2}+h_{03} h_{2}^{3}+h_{2}^{4}-5 h_{3}^{4}\right)\right) c_{3} \text {, } \\
& r_{9}=\left(-\frac{1}{15}\left(-h_{01}^{3}-2 h_{01}^{2} h_{1}-3 h_{01} h_{1}^{2}+6 h_{1}^{3}\right) b_{02}^{2}+\frac{1}{5} b_{01}\left(h_{01}^{2}+\frac{4}{3} h_{01} h_{1}+h_{1}^{2}\right)\left(h_{01}-h_{1}\right) b_{02}-\right. \\
& \left.-\frac{4}{15} b_{01}^{2}\left(h_{01}^{3}+\frac{3}{4} h_{01}^{2} h_{1}+\frac{1}{2} h_{01} h_{1}^{2}+\frac{1}{4} h_{1}^{3}\right)\right) c_{1}-\frac{2}{3}\left(h_{2}^{3}-h_{1}^{3}\right) b_{02}^{2} c_{2}- \\
& -\left(\frac{1}{15}\left(h_{03}-h_{2}\right)\left(h_{03}^{2}+3 h_{03} h_{2}+6 h_{2}^{2}\right) b_{02}^{2}+\frac{1}{5}\left(h_{03}-h_{2}\right) b_{02} b_{03}\left(h_{03}^{2}+\frac{4}{3} h_{03} h_{2}+h_{2}^{2}\right)-\right. \\
& \left.-\frac{4}{15} b_{03}^{2}\left(h_{03}^{3}+\frac{3}{4} h_{03}^{2} h_{2}+\frac{1}{2} h_{03} h_{2}^{2}+\frac{1}{4} h_{2}^{3}-\frac{5}{2} h_{3}^{3}\right)\right) c_{3},
\end{aligned}
$$




$$
\begin{gathered}
r_{10}=\left(\frac{1}{10}\left(h_{1}-h_{01}\right) b_{02}^{4}-\frac{1}{10}\left(h_{01}-h_{1}\right) b_{01} b_{02}^{3}-\frac{1}{10}\left(h_{01}-h_{1}\right) b_{01}^{2} b_{02}^{2}-\right. \\
\left.-\frac{1}{10}\left(h_{01}-h_{1}\right) b_{02} b_{01}^{3}+\frac{2}{5}\left(h_{01}+\frac{1}{4} h_{1}\right) b_{01}^{4}\right) c_{1}+\frac{1}{2}\left(h_{2}-h_{1}\right) b_{02}^{4} c_{2}+ \\
+\left(\frac{1}{10}\left(h_{03}-h_{2}\right) b_{02}^{4}+\frac{1}{10} b_{03}\left(h_{03}-h_{2}\right) b_{02}^{3}+\right. \\
\left.+\frac{1}{10}\left(h_{03}-h_{2}\right) b_{02}^{2} b_{03}^{2}+\frac{1}{10}\left(h_{03}-h_{2}\right) b_{02} b_{03}^{3}-\frac{2}{5}\left(h_{03}+\frac{1}{4} h_{2}-\frac{5}{4} h_{3}\right) b_{03}^{4}\right) c_{3} .
\end{gathered}
$$

From fastening conditions of the rod, we have

$$
\begin{gathered}
w_{0}^{(0)}(0)=w_{0}^{(0)}(l)=v_{0}^{(0)}(0)=v_{0}^{(0)}(l)=u_{0}^{(0)}(0)=u_{0}^{(0)}(l)=0, \\
\left.\frac{d v_{0}^{(0)}}{d x}\right|_{x=0}=\left.\frac{d v_{0}^{(0)}}{d x}\right|_{x=l}=\left.\frac{d w_{0}^{(0)}}{d x}\right|_{x=0}=\left.\frac{d w_{0}^{(0)}}{d x}\right|_{x=l}=0 .
\end{gathered}
$$

Then the ratios (24), (25) have the form

$$
\frac{d^{4} w_{0}^{(0)}}{d x^{4}}=\alpha_{3}+\alpha_{4} x, \frac{d^{4} v_{0}^{(0)}}{d x^{4}}=\alpha_{5}+\alpha_{6} x,
$$

where

$$
\begin{aligned}
& \alpha_{3}=\frac{1}{l}\left(g_{4} q_{0 x}+g_{5} m_{0 z}+g_{6} m_{0 y}\right)-g_{5} q_{o y} \alpha_{1}-g_{6} q_{o z} \beta_{1}, \quad \alpha_{4}=-g_{5} \alpha_{2} q_{o y}-g_{6} \beta_{2} q_{0 z}, \\
& \alpha_{5}=\frac{1}{l}\left(g_{7} q_{0 x}+g_{8} m_{0 z}+g_{9} m_{0 y}\right)-g_{8} q_{o y} \alpha_{1}-g_{9} q_{o z} \beta_{1}, \quad \alpha_{6}=-g_{8} \alpha_{2} q_{o y}-g_{9} \beta_{2} q_{0 z} .
\end{aligned}
$$

Integrating the ratios (42), we obtain

$$
\begin{aligned}
& w_{0}^{(0)}=\alpha_{4} \frac{x^{5}}{120}+\alpha_{3} \frac{x^{4}}{24}+C_{1} x^{3}+C_{2} x^{2}+C_{3} x+C_{4}, \\
& v_{0}^{(0)}=\alpha_{6} \frac{x^{5}}{120}+\alpha_{5} \frac{x^{4}}{24}+C_{5} x^{3}+C_{6} x^{2}+C_{7} x+C_{8} .
\end{aligned}
$$

From the ratios (26), (43), (44) we obtain

$$
u_{0}^{(0)}=\alpha_{7} \frac{x^{4}}{12}+\alpha_{8} \frac{x^{3}}{6}-\left(\frac{f_{3}}{f_{1}} C_{1}+\frac{f_{2}}{f_{1}} C_{5}\right) \frac{x^{2}}{2}+C_{9} x+C_{10},
$$

where

$$
\alpha_{7}=-\frac{f_{3}}{f_{1}} \frac{\alpha_{4}}{2}-\frac{f_{2}}{f_{1}} \frac{\alpha_{6}}{2}, \alpha_{8}=-\frac{q_{0}}{l f_{1}}-\frac{f_{3}}{f_{1}} \alpha_{3}-\frac{f_{2}}{f_{1}} \alpha_{5} .
$$

From (43), (44), (45) and expression (21) we obtain

$$
\begin{gathered}
N^{(0)}=\left(\frac{f_{1} \alpha_{7}}{3}+\frac{f_{2} \alpha_{6}}{6}+\frac{f_{3} \alpha_{4}}{6}\right) x^{3}+\left(\frac{f_{1} \alpha_{8}}{2}+\frac{f_{2} \alpha_{5}}{2}+\frac{f_{3} \alpha_{3}}{2}\right) x^{2}+ \\
+C_{9} f_{1}+C_{6} f_{2}+C_{2} f_{3}, \\
M_{y}^{(0)}=\left(\frac{f_{3} \alpha_{7}}{3}+\frac{f_{4} \alpha_{6}}{6}+\frac{f_{5} \alpha_{4}}{6}\right) x^{3}+\left(\frac{f_{3} \alpha_{8}}{2}+\frac{f_{4} \alpha_{5}}{2}+\frac{f_{5} \alpha_{3}}{2}\right) x^{2}+ \\
+\left(C_{1}\left(f_{5}-f_{3}^{2} f_{1}\right)+C_{5}\left(f_{4}-\frac{f_{2} f_{3}}{f_{1}}\right)\right) x+C_{9} f_{3}+C_{6} f_{4}+C_{2} f_{5},
\end{gathered}
$$




$$
\begin{aligned}
& M_{z}^{(0)}=\left(\frac{f_{2} \alpha_{7}}{3}+\frac{f_{5} \alpha_{6}}{6}+\frac{f_{6} \alpha_{4}}{6}\right) x^{3}+\left(\frac{f_{2} \alpha_{8}}{2}+\frac{f_{5} \alpha_{5}}{2}+\frac{f_{6} \alpha_{3}}{2}\right) x^{2}+ \\
& +\left(C_{1}\left(f_{6}-\frac{f_{3} f_{2}}{f_{1}}\right)+C_{5}\left(f_{5}-\frac{f_{2}^{2}}{f_{1}}\right)\right) x+C_{9} f_{2}+C_{6} f_{5}+C_{2} f_{6},
\end{aligned}
$$

From fastening conditions (40) we have

$$
\begin{gathered}
C_{3}=C_{4}=C_{7}=C_{8}=C_{10}=0, \\
C_{1}=\frac{\alpha_{8}}{l^{2}}-2 \frac{\alpha_{7}}{l^{3}}, \quad C_{2}=3 \frac{\alpha_{7}}{l^{2}}-\frac{\alpha_{8}}{l}, \quad C_{5}=\frac{\alpha_{10}}{l^{2}}-2 \frac{\alpha_{9}}{l^{3}}, \quad C_{6}=3 \frac{\alpha_{9}}{l^{2}}-\frac{\alpha_{10}}{l}, \\
C_{9}=-\alpha_{7} \frac{l^{3}}{12}-\alpha_{8} \frac{l^{2}}{6}+\left(\frac{f_{3}}{f_{1}} C_{1}+\frac{f_{2}}{f_{1}} C_{5}\right) \frac{l}{2} .
\end{gathered}
$$

where

$$
\alpha_{7}=-\alpha_{4} \frac{l^{5}}{120}-\alpha_{3} \frac{l^{4}}{24}, \quad \alpha_{8}=-\alpha_{4} \frac{l^{4}}{24}-\alpha_{3} \frac{l^{3}}{6}, \quad \alpha_{9}=-\alpha_{6} \frac{l^{5}}{120}-\alpha_{5} \frac{l^{4}}{24}, \quad \alpha_{10}=-\alpha_{6} \frac{l^{4}}{24}-\alpha_{5} \frac{l^{3}}{6},
$$

Thus, the movements in the zero approximation are completely determined.

$$
\begin{gathered}
u_{0}^{(0)}=\alpha_{7} \frac{x^{4}}{12}+\alpha_{8} \frac{x^{3}}{6}-\left(\frac{f_{3}}{f_{1}} C_{1}+\frac{f_{2}}{f_{1}} C_{5}\right) \frac{x^{2}}{2}+C_{9} x, \\
v_{0}^{(0)}=\alpha_{6} \frac{x^{5}}{120}+\alpha_{5} \frac{x^{4}}{24}+C_{5} x^{3}+C_{6} x^{2}, \\
w_{0}^{(0)}=\alpha_{4} \frac{x^{5}}{120}+\alpha_{3} \frac{x^{4}}{24}+C_{1} x^{3}+C_{2} x^{2} .
\end{gathered}
$$

From (29), (30), (31), (46), (47), (48) we obtain

$$
j_{1}(x)=\alpha_{11} x^{6}+\alpha_{12} x^{5}+\alpha_{13} x^{4}+\alpha_{14} x^{3}+\alpha_{15} x^{2}+\alpha_{16} x+\alpha_{17}
$$

where

$$
\begin{gathered}
\alpha_{11}=\frac{1}{36} \alpha_{4}^{2} r_{6}+\frac{1}{18}\left(\alpha_{6} r_{5}+2 \alpha_{7} r_{3}\right) \alpha_{4}+\frac{1}{9} \alpha_{7}^{2} r_{1}+\frac{1}{9} \alpha_{6} \alpha_{7} r_{2}+\frac{1}{36} \alpha_{6}^{2} r_{4}, \\
\alpha_{12}=\frac{1}{6}\left(\alpha_{4} r_{6}+\alpha_{6} r_{5}+2 \alpha_{7} r_{3}\right) \alpha_{3}+\frac{1}{6}\left(\alpha_{5} r_{5}+\alpha_{8} r_{3}\right) \alpha_{4}+\frac{1}{6}\left(\alpha_{6} r_{4}+2 \alpha_{7} r_{2}\right) \alpha_{5}+ \\
+\frac{1}{3} r_{1} \alpha_{7} \alpha_{8}+\frac{1}{6} \alpha_{8} r_{2} \alpha_{6} \\
\alpha_{13}=\frac{1}{12} \frac{1}{f_{1}}\left(\left(24 \alpha_{4} r_{6}+24 \alpha_{6} r_{5}+48 \alpha_{7} r_{3}\right) C_{1}+\left(24 \alpha_{4} r_{5}+24 \alpha_{6} r_{4}+48 \alpha_{7} r_{2}\right) C_{5}+6 r_{5} \alpha_{5} \alpha_{3}+\right. \\
\left.\left.+3 r_{6} \alpha_{3}^{2}+6 \alpha_{3} \alpha_{8} r_{3}+3 \alpha_{5}^{2} r_{4}+6 \alpha_{5} \alpha_{8} r_{2}+3 \alpha_{8}^{2} r_{1}\right) f_{1}-4\left(\alpha_{4} r_{3}+\alpha_{6} r_{2}+2 \alpha_{7} r_{1}\right)\left(C_{1} f_{3}+C_{5} f_{2}\right)\right), \\
\alpha_{14}=\frac{1}{3 f_{1}}\left(\left(\left(18 \alpha_{3} r_{6}+18 \alpha_{5} r_{5}+18 \alpha_{8} r_{3}\right) C_{1}+\left(18 \alpha_{3} r_{5}+18 \alpha_{5} r_{4}+18 \alpha_{8} r_{2}\right) C_{5}+\right.\right. \\
+\left(\alpha_{6} C_{9}+4 \alpha_{7} C_{6}\right) r_{2}+\left(\alpha_{4} C_{9}+4 \alpha_{7} C_{2}\right) r_{3}+\left(2 \alpha_{4} C_{6}+2 \alpha_{6} C_{2}\right) r_{5}+2 r_{4} \alpha_{6} C_{6}+ \\
\left.\left.+2 r_{6} \alpha_{4} C_{2}+2 \alpha_{7} C_{9} r_{1}\right) f_{1}-3\left(\alpha_{3} r_{3}+\alpha_{5} r_{2}+\alpha_{8} r_{1}\right)\left(C_{1} f_{3}+C_{5} f_{2}\right)\right) \\
\alpha_{15}=\frac{1}{f_{1}^{2}}\left(\left(36 C_{1}^{2} r_{6}+72 r_{5} C_{5} C_{1}+36 r_{4} C_{5}^{2}+\alpha_{8} C_{9} r_{1}+\left(\alpha_{5} C_{9}+2 \alpha_{8} C_{6}\right) r_{2}+\right.\right. \\
\left.+\left(\alpha_{3} C_{9}+2 \alpha_{8} C_{2}\right) r_{3}+\left(2 C_{2} r_{6}+2 C_{6} r_{5}\right) \alpha_{3}+2 \alpha_{5}\left(C_{2} r_{5}+C_{6} r_{4}\right)\right) f_{1}^{2}- \\
\left.-12\left(C_{1} f_{3}+C_{5} f_{2}\right)\left(C_{1} r_{3}+C_{5} r_{2}\right) f_{1}+r_{1}\left(C_{1} f_{3}+C_{5} f_{2}\right)^{2}\right)
\end{gathered}
$$




$$
\begin{gathered}
\alpha_{16}=\frac{1}{f_{1}}\left(\left(\left(24 C_{2} r_{6}+24 C_{6} r_{5}+12 C_{9} r_{3}\right) C_{1}+12 C_{5}\left(2 C_{2} r_{5}+2 C_{6} r_{4}+C_{9} r_{2}\right)\right) f_{1}-\right. \\
\left.-\left(C_{1} f_{3}+C_{5} f_{2}\right)\left(4 r_{2} C_{6}+4 r_{3} C_{2}+2 r_{1} C_{9}\right)\right), \\
\alpha_{17}=C_{9}^{2} r_{1}+\left(4 C_{2} r_{3}+4 C_{6} r_{2}\right) C_{9}+4 C_{2}^{2} r_{6}+8 C_{2} C_{6} r_{5}+4 C_{6}^{2} r_{4}, \\
j_{2}(x)=\alpha_{18} x^{6}+\alpha_{19} x^{5}+\alpha_{20} x^{4}+\alpha_{21} x^{3}+\alpha_{22} x^{2}+\alpha_{23} x+\alpha_{24},
\end{gathered}
$$

where

$$
\begin{aligned}
& \alpha_{18}=\frac{1}{36} \alpha_{4}^{2} r_{8}+\frac{1}{18}\left(\alpha_{6} r_{9}+2 \alpha_{7} r_{5}\right) \alpha_{4}+\frac{1}{9} \alpha_{7}^{2} r_{2}+\frac{1}{9} \alpha_{6} \alpha_{7} r_{4}+\frac{1}{36} \alpha_{6}^{2} r_{7} \\
& \alpha_{19}=\frac{1}{6}\left(\alpha_{4} r_{8}+\alpha_{6} r_{9}+2 \alpha_{7} r_{5}\right) \alpha_{3}+\frac{1}{6}\left(\alpha_{5} r_{9}+\alpha_{8} r_{5}\right) \alpha_{4}+\frac{1}{6}\left(\alpha_{6} r_{7}+2 \alpha_{7} r_{4}\right) \alpha_{5}+ \\
& +\frac{1}{3} r_{2} \alpha_{7} \alpha_{8}+\frac{1}{6} \alpha_{6} \alpha_{8} r_{4} \\
& \alpha_{20}=\frac{1}{12 f_{1}}\left(\left(\left(24 \alpha_{4} r_{8}+24 \alpha_{6} r_{9}+48 \alpha_{7} r_{5}\right) C_{1}+\left(24 \alpha_{4} r_{9}+24 \alpha_{6} r_{7}+48 \alpha_{7} r_{4}\right) C_{5}+\right.\right. \\
& \left.+3 r_{8} \alpha_{3}^{2}+6 r_{9} \alpha_{5} \alpha_{3}+6 \alpha_{3} \alpha_{8} r_{5}+3 r_{7} \alpha_{5}^{2}+6 \alpha_{5} \alpha_{8} r_{4}+3 r_{2} \alpha_{8}^{2}\right) f_{1}- \\
& \left.-4\left(\alpha_{4} r_{5}+\alpha_{6} r_{4}+2 \alpha_{7} r_{2}\right)\left(C_{1} f_{3}+C_{5} f_{2}\right)\right) \\
& \alpha_{21}=\frac{1}{3 f_{1}}\left(\left(\left(18 \alpha_{3} r_{8}+18 \alpha_{5} r_{9}+18 \alpha_{8} r_{5}\right) C_{1}+\left(18 \alpha_{3} r_{9}+18 \alpha_{5} r_{7}+18 \alpha_{8} r_{4}\right) C_{5}+\right.\right. \\
& +\left(\alpha_{6} C_{9}+4 \alpha_{7} C_{6}\right) r_{4}+\left(\alpha_{4} C_{9}+4 \alpha_{7} C_{2}\right) r_{5}+\left(2 \alpha_{4} C_{6}+2 \alpha_{6} C_{2}\right) r_{9}+2 r_{7} C_{6} \alpha_{6}+ \\
& \left.\left.2 r_{8} C_{2} \alpha_{4}+2 r 2 \alpha_{7} C_{9}\right) f_{1}-3\left(\alpha_{3} r_{5}+\alpha_{5} r_{4}+\alpha_{8} r_{2}\right)\left(C_{1} f_{3}+C_{5} f_{2}\right)\right), \\
& \alpha_{22}=\frac{1}{f_{1}^{2}}\left(\left(36 r_{8} C_{1}^{2}+72 r_{9} C_{5} C_{1}+36 r_{7} C_{5}^{2}+r_{2} \alpha_{8} C_{9}+\left(\alpha_{5} C_{9}+2 \alpha_{8} C_{6}\right) r_{4}+\right.\right. \\
& +\left(\alpha_{3} C_{9}+2 \alpha_{8} C_{2}\right) r_{5}+\left(\left(2 C_{2} r_{8}+2 C_{6} r_{9}\right) \alpha_{3}+2 \alpha_{5}\left(C_{2} r_{9}+C_{6} r_{7}\right)\right) f_{1}^{2}- \\
& \left.-12\left(C_{1} f_{3}+C_{5} f_{2}\right)\left(C_{1} r_{5}+C_{5} r_{4}\right) f_{1}+r_{2}\left(C_{1} f_{3}+C_{5} f_{2}\right)^{2}\right), \\
& \alpha_{23}=\frac{1}{f_{1}}\left(\left(\left(864 C_{2} r_{8}+864 C_{6} r_{9}+432 C_{9} r_{5}\right) C_{1}+432 C_{5}\left(2 C_{2} r_{9}+2 C_{6} r_{7}+C_{9} r_{4}\right)\right) f_{1}^{3}-\right. \\
& \left.-\left(C_{1} f_{3}+C_{5} f_{2}\right)\left(4 C_{2} r_{5}+C_{6} r_{4}+2 C_{9} r_{2}\right)\right) \\
& \alpha_{24}=4 C_{2}^{2} r_{8}+8 C_{2} C_{6} r_{9}+4 C_{2} C_{9} r_{5}+4 C_{6}^{2} r_{7}+4 C_{6} C_{9} r_{4}+C_{9}^{2} r_{2} \text {, } \\
& j_{3}(x)=\alpha_{25} x^{6}+\alpha_{26} x^{5}+\alpha_{27} x^{4}+\alpha_{28} x^{3}+\alpha_{29} x^{2}+\alpha_{30} x+\alpha_{31},
\end{aligned}
$$

where

$$
\begin{gathered}
\alpha_{25}=\frac{1}{36} \alpha_{4}^{2} r_{10}+\frac{1}{72}\left(4 \alpha_{6} r_{8}+8 \alpha_{7} r_{6}\right) \alpha_{4}+\frac{1}{9} \alpha_{7}^{2} r_{3}+\frac{1}{9} \alpha_{6} \alpha_{7} r_{5}+\frac{1}{72} \alpha_{6}^{2} r_{9}, \\
\alpha_{26}=\frac{1}{6}\left(\alpha_{4} r_{10}+\alpha_{6} r_{8}+2 \alpha_{7} r_{6}\right) \alpha_{3}+\frac{1}{6}\left(\alpha_{5} r_{8}+\alpha_{8} r_{6}\right) \alpha_{4}+\frac{1}{12}\left(\alpha_{6} r_{9}+4 \alpha_{7} r_{5}\right) \alpha_{5}+ \\
+\frac{1}{3} r_{3} \alpha_{7} \alpha_{8}+\frac{1}{6} \alpha_{6} \alpha_{8} r_{5}, \\
\alpha_{27}=\frac{1}{24 f_{1}}\left(\left(\left(48 \alpha_{4} r_{10}+48 \alpha_{6} r_{8}+96 \alpha_{7} r_{6}\right) C_{1}+\left(48 \alpha_{4} r_{8}+24 \alpha_{6} r_{9}+96 \alpha_{7} r_{5}\right) C_{5}+3 r_{9} \alpha_{5}^{2}+\right.\right. \\
\left.+6 r_{10} \alpha_{3}^{2}+12 r_{8} \alpha_{5} \alpha_{3}+12 r_{6} \alpha_{3} \alpha_{8}+12 r_{5} \alpha_{5} \alpha_{8}+6 r_{3} \alpha_{8}^{2}\right) f_{1}- \\
\left.-\left(\alpha_{4} r_{6}+\alpha_{6} r_{5}+2 \alpha_{7} r_{3}\right)\left(C_{1} f_{3}+C_{5} f_{2}\right)\right),
\end{gathered}
$$




$$
\begin{aligned}
& \alpha_{28}=\frac{1}{3 f_{1}}\left(\left(\left(18 \alpha_{3} r_{10}+18 \alpha_{5} r_{8}+18 \alpha_{8} r_{6}\right) C_{1}+\left(18 \alpha_{3} r_{8}+9 \alpha_{5} r_{9}+18 \alpha_{8} r_{5}\right) C_{5}+\right.\right. \\
& +\left(\alpha_{6} C_{9}+4 \alpha_{7} C_{6}\right) r_{5}+\left(\alpha_{4} C_{9}+4 \alpha_{7} C_{2}\right) r_{6}+\left(2 \alpha_{4} C_{6}+2 \alpha_{6} C_{2}\right) r_{8}+r_{9} C_{6} \alpha_{6}+2 r_{10} C_{2} \alpha_{4}+ \\
& \left.\left.+2 r_{3} \alpha_{7} C_{9}\right) f_{1}-3\left(\alpha_{3} r_{6}+\alpha_{5} r_{5}+\alpha_{8} r_{3}\right)\left(C_{1} f_{3}+C_{5} f_{2}\right)\right) \text {, } \\
& \alpha_{29}=\frac{1}{f_{1}^{2}}\left(\left(36 r_{10} C_{1}^{2}+72 r_{8} C_{5} C_{1}+18 r_{9} C_{5}^{2}+r_{3} \alpha_{8} C_{9}+\left(\alpha_{5} C_{9}+2 \alpha_{8} C_{6}\right) r_{5}+\right.\right. \\
& \left.+\left(\alpha_{3} C_{9}+2 \alpha_{8} C_{2}\right) r_{6}+\left(2 C_{2} r_{10}+2 C_{6} r_{8}\right) \alpha_{3}+\alpha_{5}\left(2 C_{2} r_{8}+C_{6} r_{9}\right)\right) f_{1}^{2}- \\
& \left.-12\left(C_{1} f_{3}+C_{5} f_{2}\right)\left(C_{1} r_{6}+C_{5} r_{5}\right) f_{1}+r_{3}\left(C_{1} f_{3}+C_{5} f_{2}\right)^{2}\right) \text {, } \\
& \alpha_{30}=\frac{1}{f_{1}}\left(\left(\left(24 C_{2} r_{10}+24 C_{6} r_{8}+12 C_{9} r_{6}\right) C_{1}+12 C_{5}\left(2 C_{2} r_{8}+C_{6} r_{9}+C_{9} r_{5}\right)\right) f_{1}-\right. \\
& \left.-\left(C_{1} f_{3}+C_{5} f_{2}\right)\left(4 C_{6} r_{5}+4 C_{2} r_{6}+2 C_{9} r_{3}\right)\right), \\
& \alpha_{31}=C_{9}^{2} r_{3}+\left(4 C_{2} r_{6}+4 C_{6} r_{5}\right) C_{9}+4 C_{2}^{2} r_{10}+8 C_{2} C_{6} r_{8}+2 C_{6}^{2} r_{9} .
\end{aligned}
$$

Substituting the ratios (49), (50), (51) in expressions (34), (35), (36), we get the differential equations to determine the movements in the first approximation

$$
\begin{gathered}
\frac{d^{4} v_{0}^{(1)}}{d x^{4}}=\alpha_{32} x^{4}+\alpha_{33} x^{3}+\alpha_{34} x^{2}+\alpha_{35} x+\alpha_{36}, \\
\frac{d^{4} w_{0}^{(1)}}{d x^{4}}=\alpha_{37} x^{4}+\alpha_{38} x^{3}+\alpha_{39} x^{2}+\alpha_{40} x+\alpha_{41}, \\
\frac{d^{2} u_{0}^{(1)}}{d x^{2}}=\alpha_{42} x^{5}+\alpha_{43} x^{4}+\alpha_{44} x^{3}+\alpha_{45} x^{2}+\alpha_{46} x+\alpha_{47},
\end{gathered}
$$

where

$$
\begin{gathered}
\alpha_{32}=30\left(\alpha_{11} r_{12}+\alpha_{18} r_{13}+\alpha_{25} r_{14}\right), \quad \alpha_{33}=20\left(\alpha_{12} r_{12}+\alpha_{19} r_{13}+\alpha_{26} r_{14}\right), \\
\alpha_{34}=12\left(\alpha_{13} r_{12}+\alpha_{20} r_{13}+\alpha_{27} r_{14}\right), \quad \alpha_{35}=6\left(\alpha_{14} r_{12}+\alpha_{21} r_{13}+\alpha_{28} r_{14}\right), \\
\alpha_{36}=2\left(\alpha_{15} r_{12}+\alpha_{22} r_{13}+\alpha_{29} r_{14}\right), \quad \alpha_{37}=30\left(\alpha_{11} r_{15}+\alpha_{18} r_{16}+\alpha_{25} r_{17}\right), \\
\alpha_{38}=20\left(\alpha_{12} r_{15}+\alpha_{19} r_{16}+\alpha_{26} r_{17}\right), \quad \alpha_{39}=12\left(\alpha_{13} r_{15}+\alpha_{20} r_{16}+\alpha_{27} r_{17}\right), \\
\alpha_{40}=6\left(\alpha_{14} r_{15}+\alpha_{21} r_{16}+\alpha_{28} r_{17}\right), \quad \alpha_{41}=2\left(\alpha_{15} r_{15}+\alpha_{22} r_{16}+\alpha_{29} r_{17}\right) . \\
\alpha_{42}=-\frac{6 \alpha_{11}}{f_{1}}, \quad \alpha_{43}=-\frac{5 \alpha_{12}}{f_{1}}, \quad \alpha_{44}=-\frac{4 \alpha_{13}}{f_{1}}, \quad \alpha_{45}=-\frac{3 \alpha_{14}}{f_{1}}, \\
\alpha_{46}=-\frac{1}{f_{1}}\left(2 \alpha_{15}+720\left(f_{2}\left(r_{12} \alpha_{11}+r_{13} \alpha_{18}+r_{14} \alpha_{25}\right)+f_{3}\left(r_{15} \alpha_{11}+r_{16} \alpha_{18}+r_{17} \alpha_{25}\right)\right)\right), \\
\alpha_{47}=-\frac{1}{f_{1}}\left(\alpha_{16}+120\left(f_{2}\left(r_{12} \alpha_{12}+r_{13} \alpha_{19}+r_{14} \alpha_{26}\right)+f_{3}\left(r_{15} \alpha_{12}+r_{16} \alpha_{19}+r_{17} \alpha_{26}\right)\right)\right) .
\end{gathered}
$$

Integrating the equations (52), (53), (54), taking into account the initial conditions for the first approximation

$$
\begin{gathered}
w_{0}^{(1)}(0)=w_{0}^{(1)}(l)=v_{0}^{(1)}(0)=v_{0}^{(1)}(l)=u_{0}^{(1)}(0)=u_{0}^{(1)}(l)=0, \\
\left.\frac{d v_{0}^{(1)}}{d x}\right|_{x=0}=\left.\frac{d v_{0}^{(1)}}{d x}\right|_{x=l}=\left.\frac{d w_{0}^{(1)}}{d x}\right|_{x=0}=\left.\frac{d w_{0}^{(1)}}{d x}\right|_{x=l}=0,
\end{gathered}
$$


we get

$$
\begin{gathered}
v_{0}^{(1)}=\alpha_{32} \frac{x^{8}}{1680}+\alpha_{33} \frac{x^{7}}{840}+\alpha_{34} \frac{x^{6}}{360}+\alpha_{35} \frac{x^{5}}{120}+\alpha_{36} \frac{x^{4}}{24}+C_{11} x^{3}+C_{12} x^{2}, \\
w_{0}^{(1)}=\alpha_{37} \frac{x^{8}}{1680}+\alpha_{38} \frac{x^{7}}{840}+\alpha_{39} \frac{x^{6}}{360}+\alpha_{40} \frac{x^{5}}{120}+\alpha_{41} \frac{x^{4}}{24}+C_{13} x^{3}+C_{14} x^{2}, \\
u_{0}^{(1)}=\alpha_{42} \frac{x^{7}}{42}+\alpha_{43} \frac{x^{6}}{30}+\alpha_{44} \frac{x^{5}}{20}+\alpha_{45} \frac{x^{4}}{12}+\alpha_{46} \frac{x^{3}}{6}+\alpha_{47} \frac{x^{2}}{2}+C_{15} x,
\end{gathered}
$$

where

$$
\begin{gathered}
C_{11}=\frac{1}{l^{2}} \alpha_{48}-\frac{2}{l^{3}} \alpha_{49}, \quad C_{12}=\frac{3}{l^{2}} \alpha_{49}-\frac{1}{l} \alpha_{48}, \quad C_{13}=\frac{1}{l^{2}} \alpha_{50}-\frac{2}{l^{3}} \alpha_{51}, \quad C_{14}=\frac{3}{l^{2}} \alpha_{51}-\frac{1}{l} \alpha_{50}, \\
C_{15}=-\alpha_{42} \frac{l^{6}}{42}-\alpha_{43} \frac{l^{5}}{30}-\alpha_{44} \frac{l^{4}}{20}-\alpha_{45} \frac{l^{3}}{12}-\alpha_{46} \frac{l^{2}}{6}-\alpha_{47} \frac{l}{2}, \\
\alpha_{48}=-\alpha_{32} \frac{l^{7}}{210}-\alpha_{33} \frac{l^{6}}{140}-\alpha_{34} \frac{l^{5}}{60}-\alpha_{35} \frac{l^{4}}{24}-\alpha_{36} \frac{l^{3}}{6}, \\
\alpha_{49}=-\alpha_{32} \frac{l^{8}}{1680}-\alpha_{33} \frac{l^{7}}{840}-\alpha_{34} \frac{l^{6}}{360}-\alpha_{35} \frac{l^{5}}{120}-\alpha_{36} \frac{l^{4}}{24}, \\
\alpha_{50}=-\alpha_{37} \frac{l^{7}}{210}-\alpha_{38} \frac{l^{6}}{140}-\alpha_{39} \frac{l^{5}}{60}-\alpha_{40} \frac{l^{4}}{24}-\alpha_{41} \frac{l^{3}}{6}, \\
\alpha_{51}=-\alpha_{37} \frac{l^{8}}{1680}-\alpha_{38} \frac{l^{7}}{840}-\alpha_{39} \frac{l^{6}}{360}-\alpha_{40} \frac{l^{5}}{120}-\alpha_{41} \frac{l^{4}}{24} .
\end{gathered}
$$

The expression for longitudinal force and bending moments in the first approximation can be obtained from (29), (30), (31), (49), (50), (51), (57), (58), (59).

\section{Discussion}

Thus, the problem of complex bend of the rod is solved by the method of small parameter in the zero and first approximations. This method can be used to solve the problem in the second and subsequent approximations.

This work is carried out with the partial financial support of RFBR grant (project number 19-01-00038).

\section{References}

[1] A.V. Mishenko, Y.V. Nemirovskii, Izvestiya vuzov. Stroitelstvo pp. 5-12 (2013)

[2] Y.V. Nemirovskii, A.V. Mishenko, R.F. Terletskii, Journal of Mathematical Sciences 223, 87 (2017)

[3] N.V. Minaeva, A.A. Sizikova, Vestnik Chuvashskogo Gosudarstvennogo Pedagogicheskogo Universiteta im. I. Ya. Yakovleva. Seriya: Mekhanika Predelnogo Sostoyaniya pp. 125-132 (2017)

[4] Y.V. Nemirovskii, S.V. Tikhonov, MATEC Web of Conferences 196, 01038 (2018)

[5] V.P. Pikulin, S.I. Pohozaev, Equations in Mathematical Physics: A practical course (MCNMO, Moscow, 2004) 
[6] D.D. Ivlev, L.V. Ershov, Perturbation Method in the Theory of Elastoplastic Bodies (Nauka, Moscow, 1978)

[7] A.A. Ilyushin, in Engineering Digest (1954), Vol. 19

[8] O.D. Gorbenko, N.V. Minaeva, in Improving Ground Support for Aviation (Voronezh, 2000), Vol. 1

[9] N.V. Minaeva, Izvestiya RAN. Mekhanika Tverdogo Tela pp. 37-39 (2008)

[10] Y.V. Nemirovskii, Vestnik Chuvashskogo Gosudarstvennogo Pedagogicheskogo Universiteta im. I. Ya. Yakovleva. Seriya: Mekhanika Predelnogo Sostoyaniya pp. 26-37 (2018)

[11] L.R. Mailyan, E.I. Ivashchenko, Calculation of Reinforced Concrete Elements Based on Actual Material Strain Diagrams (Rostov State University of Civil Engineering, Rostov-on-Don, 2006)

[12] A.G. Gorshkov, V.N. Troshin, V.I. Shalashilin, Strength of Materials (Fizmatlit, Moscow, 2005) 